University of Nebraska - Lincoln

DigitalCommons@University of Nebraska - Lincoln

Michigan Bovine Tuberculosis Bibliography and

Database

Wildlife Disease and Zoonotics

$5-2001$

\title{
Experimental Deer-To-Deer Transmission of Mycobacterium bovis
}

\author{
Mitchell V. Palmer \\ USDA-ARS, Mitchell.Palmer@ars.usda.gov
}

Diana L. Whipple

W. Ray Waters

Follow this and additional works at: https://digitalcommons.unl.edu/michbovinetb

Part of the Veterinary Medicine Commons

Palmer, Mitchell V.; Whipple, Diana L.; and Waters, W. Ray, "Experimental Deer-To-Deer Transmission of Mycobacterium bovis" (2001). Michigan Bovine Tuberculosis Bibliography and Database. 79. https://digitalcommons.unl.edu/michbovinetb/79

This Article is brought to you for free and open access by the Wildlife Disease and Zoonotics at DigitalCommons@University of Nebraska - Lincoln. It has been accepted for inclusion in Michigan Bovine Tuberculosis Bibliography and Database by an authorized administrator of DigitalCommons@University of Nebraska Lincoln. 


\title{
Experimental deer-to-deer transmission of Mycobacterium bovis
}

\author{
Mitchell V. Palmer, DVM, PhD; Diana L. Whipple, MS, and W. Ray Waters, DVM, PhD
}

\begin{abstract}
Objective-To determine whether Mycobacterium bovis can be transmitted from experimentally infected deer to uninfected in-contact deer.
\end{abstract}

Animals-Twenty-three 6-month-old white-tailed deer.

Procedure-On day $0, M$ bovis $\left(2 \times 10^{8}\right.$ colony-forming units) was administered by intratonsillar instillation to 8 deer; 3 control deer received saline $(0.9 \% \mathrm{NaCl})$ solution. Eight in-contact deer were comingled with inoculated deer from day 21. On day 120, inoculated deer were euthanatized and necropsied. On day 180, 4 in-contact deer were euthanatized, and 4 new incontact deer were introduced. On day 360, all in-contact deer were euthanatized. Rectal, oral, and nasal swab specimens and samples of hay, pelleted feed, water, and feces were collected for bacteriologic culture. Tissue specimens were also collected at necropsy for bacteriologic culture and histologic analysis.

Results-On day 90, inoculated and in-contact deer developed delayed-type hypersensitivity (DTH) reactions to purified protein derivative of $M$ bovis. Similarly, new in-contact deer developed DTH reactions by 100 days of contact with original in-contact deer. Tuberculous lesions in in-contact deer were most commonly detected in lungs and tracheobronchial and medial retropharyngeal lymph nodes. Mycobacterium bovis was isolated from nasal secretions and saliva from inoculated and in-contact deer, urine and feces from in-contact deer, and hay and pelleted feed.

Conclusions and Clinical Relevance-Mycobacterium bovis is efficiently transmitted from experimentally infected deer to uninfected in-contact deer through nasal secretions, saliva, or contaminated feed. Wildlife management practices that result in unnatural gatherings of deer may enhance both direct and indirect transmission of $M$ bovis. (Am J Vet Res $2001 ; 62: 692-696)$

Tn 1994, tuberculosis attributable to infection with Mycobacterium bovis was diagnosed in a free-ranging white-tailed deer (Odocoileus virginianus) in Michigan. ${ }^{1}$ Subsequent surveys conducted by the Michigan Department of Natural Resources and the Michigan State University Animal Health Diagnostic Laboratory

Received Apr 24, 2000.

Accepted Jun 26, 2000

From the Bacterial Diseases of Livestock Research Unit, National Animal Disease Center, Agricultural Research Service, USDA, 2300 Dayton Ave, Ames, IA 50010.

The authors thank Rebecca Lyon, Jody Mentele, Lori Dethloff, and Kevin Esch for technical assistance.

Names are necessary to report factually on available data; however, the USDA neither guarantees nor warrants the standard of the product, and the use of the name by USDA implies no approval of the product to the exclusion of others that may also be suitable. identified an epidemic of $M$ bovis infection in freeranging white-tailed deer in northeast Michigan. This ongoing epidemic represents the first known reservoir of $M$ bovis in free-ranging wildlife in the United States and the first known epidemic of tuberculosis in whitetailed deer. Several factors are thought to have contributed to the establishment and persistence of $M$ bovis in this wildlife reservoir. These factors include the large number of cattle infected with $M$ bovis in Michigan during the late 1950 s, $^{2}$ a deer population that has steadily increased beyond the capacity of the habitat (eg, focal concentrations of 19 to 23 deer $/ \mathrm{km}^{2}$ ), ${ }^{1}$ and long-term winter feeding of deer to prevent migration and decrease mortality to maintain high deer numbers for hunting purposes. ${ }^{1}$ The resulting increased population combined with prolonged crowding of deer around feeding sites provided increased opportunity for deer-to-deer contact and enhanced transmission of $M$ bovis. Results of DNA analysis of $M$ bovis isolates from Michigan white-tailed deer indicated that the majority of deer were infected with a common strain of $M$ bovis. This suggests a single source of infection. ${ }^{3}$ Infection of wildlife with $M$ bovis represents a serious threat to domestic livestock. Twelve $M$ bovis-infected cattle herds have been identified in Michigan since diagnosis of tuberculosis in white-tailed deer. Restriction fragment length polymorphism analyses indicate that $M$ bovis isolates from deer and cattle are identical, suggesting cattle became infected through contact with free-ranging white-tailed deer. ${ }^{a}$

Little is known about the susceptibility of whitetailed deer to infection with $M$ bovis, the etiopathogenesis of tuberculosis in white-tailed deer, or the ability of $M$ bovis to be transmitted from deer to deer or other susceptible hosts. The purpose of the study reported here was to determine whether $M$ bovis can be transmitted from experimentally infected deer to uninfected in-contact deer. Furthermore, routes of transmission between deer, including the role of contaminated feed and water, were investigated.

\section{Materials and Methods}

Animals and groups-Nineteen (8 castrated males, 11 sexually intact females) 6-month-old white-tailed deer were randomly assigned to 1 of the following groups: inoculated ( $\mathrm{n}=8$ females), original in-contact (8 castrated males), and control (3 females). Mycobacterium bovis strain $1315\left(2 \times 10^{8}\right.$ colony-forming units) was administered to deer in the inoculated group via intratonsillar instillation as described ${ }^{4}$ on day 0 . Mycobacterium bovis strain 1315 was originally isolated from a white-tailed deer in Michigan in 1995. Controls were inoculated with sterile saline $(0.9 \% \mathrm{NaCl})$ solution at the same time and housed separately. Twenty-one days after inoculation, original in-contact deer were introduced and housed such that 2 in-contact deer were housed with 2 inoculated deer in a pen of approximately $16 \mathrm{~m}^{2}$, resulting in a total of 4 pens. In each 
pen, deer shared a common source of water and feed. Pens were inside a biosecurity level 3 building with directional airflow such that air from the pens was pulled toward a central corridor and passed through HEPA filters before exiting the building. Airflow velocity was controlled to provide 10.4 air changes/h. Deer in each pen had access to a circulating watering device and were fed pelleted feed ${ }^{\mathrm{b}}$ and alfalfa hay. Pens were cleaned once daily with a high-pressure hose. During cleaning, deer in each pen were transferred to a holding pen and had contact with penmates only.

On day 120, inoculated deer were euthanatized and necropsied. On day 180 (159 days after introduction of original in-contact deer), 4 in-contact deer were euthanatized and necropsied. At that time, 4 age-matched female deer (new in-contact deer) were introduced to the remaining 4 incontact deer such that 2 original in-contact deer and 2 new in-contact deer were housed in each of the 2 pens previously occupied by original in-contact and inoculated deer. On day 360 (339 and 180 days after introduction of original and new in-contact deer, respectively), remaining deer, excluding controls, were euthanatized and necropsied.

Skin testing-Prior to the study and 90 days after inoculation, comparative cervical skin tests (CCT) were performed on control, inoculated, and in-contact deer as described. ${ }^{4}$ Sensitivity and specificity of the CCT in Cervidae are 90.5 and $93.4 \%$, respectively. ${ }^{5}$ Results were used to categorize deer as negative, suspect, or reactors in relation to exposure to $M$ bovis according to USDA guidelines for skin testing in Cervidae. ${ }^{6}$ New in-contact deer were tested prior to and 100 days after introduction to original in-contact deer.

Bacteriologic culture-Nasal, oral, and rectal swab specimens were collected from all deer prior to inclusion in the study; from inoculated, original in-contact, and control deer on days $21,63,90$, and 113; from control and original in-contact deer on day 150; and from control and original and new in-contact deer on days 180, 210, 240, 270, 330, and 360 . Swab specimens of the tonsillar crypt region were collected from all deer prior to the study, from inoculated deer 21 days after inoculation, and from euthanatized deer during necropsy. Tonsillar crypt specimens were collected with the aid of a laryngoscope and a sterile 18-cm cytology brush ${ }^{c}$ and processed as described. ${ }^{4}$

Samples of hay, pelleted feed, water, and feces from pen floors were collected prior to inoculation and every 30 days thereafter. Swab specimens, feed, water, and fecal samples were processed for bacteriologic culture as described. Results were considered positive when $M$ bovis was isolated.

Necropsy-Deer were euthanatized by IV injection of sodium pentobarbital. ${ }^{\mathrm{d}}$ Necropsy specimens for bacteriologic culture and histologic examination were collected from tonsil, lung, liver, spleen, kidney, brain, feces, urine, and mandibular, parotid, medial retropharyngeal, superficial cervical, tracheobronchial, mediastinal, mesenteric, hepatic, iliac, popliteal, and prefemoral lymph nodes. Additional specimens for histologic examination were collected from adrenal glands, trachea, intercostal muscle, and diaphragm.

Specimens for bacteriologic culture were placed individually in sterile bags and stored at $-80 \mathrm{C}$ until processed as described. ${ }^{8}$ Specimens for histologic examination were fixed in neutral-buffered $10 \%$ formalin and processed by routine paraffin embedment techniques. Sections were cut $5-\mu \mathrm{m}$ thick, stained with H\&E, and examined by use of light microscopy. Adjacent $5-\mu \mathrm{m}$ sections were cut from specimens with lesions suggestive of tuberculosis (eg, caseonecrotic granulomata) and stained by use of the ZiehlNeelsen technique for acid-fast bacteria. ${ }^{9}$ Microscopic findings were considered positive when lesions consistent with tuberculosis contained acid-fast bacilli.

On day 90, 1 inoculated deer was euthanatized because of an injury not related to the experimental protocol. On day 230 (209 days after introduction of original in-contact deer), 1 original in-contact deer died of tuberculosis. Thirty-nine days after introduction of new in-contact deer, 1 new in-contact deer was euthanatized because of an injury unrelated to the experimental protocol. In all cases, necropsies were performed and samples collected as described.

\section{Results}

Skin testing-Results of CCT were negative for all deer prior to inclusion in the study. On day 90 (69 days after introduction of original in-contact deer), all inoculated and original in-contact deer were classified as reactors on the basis of CCT results. One hundred days after introduction, all new in-contact deer were also classified as reactors.

Shedding of $\mathbf{M}$ bovis-Mycobacterium bovis was not isolated from swab specimens collected from any deer prior to inclusion in the study or from control deer at any time during the study. Tonsillar swab specimens from all inoculated deer contained $M$ bovis 21 days after inoculation. In addition, $M$ bovis was isolated at least once from nasal, oral, or rectal swab specimens collected from 4 of 8 inoculated deer on days 63 , 90, and 113 (Table 1). Necropsy swab specimens of the tonsillar crypt from 4 of 8 inoculated deer yielded $M$ bovis.

Mycobacterium bovis was detected in oral or nasal swab specimens from 2 of the 8 original in-contact deer 69 days after co-mingling with inoculated deer (day 90). At necropsy, tonsillar swab specimens from 1 original in-contact deer contained $M$ bovis. Mycobacterium bovis was not isolated from oral, nasal, or rectal swab specimens from new in-contact deer. However, $M$ bovis was isolated from the tonsillar crypt of 1 of 4 new in-contact deer at necropsy.

Mycobacterium bovis in feed, water, and fecesMycobacterium bovis was cultured from samples of pelleted feed collected from 1 pen on day 63 and a sepa-

Table 1-Isolation of Mycobacterium bovis from oral, nasal, tonsillar, and rectal swab specimens collected from white-tailed deer inoculated with $M$ bovis $\left(2 \times 10^{8}\right.$ colony-forming units) on day 0 or in contact with inoculated deer from day 21

\begin{tabular}{|c|c|c|c|c|c|}
\hline \multirow[b]{2}{*}{ Group } & \multicolumn{4}{|c|}{ Day after inoculation } & \multirow[b]{2}{*}{ Necropsy* } \\
\hline & 21 & 63 & 90 & 113 & \\
\hline \multicolumn{6}{|l|}{ Inoculated } \\
\hline Tonsillart & $8 / 8$ & ND & ND & ND & $4 / 8$ \\
\hline Oral & $0 / 8$ & $2 / 8$ & $1 / 8$ & $2 / 7 \ddagger$ & $0 / 8$ \\
\hline Nasal & $0 / 8$ & $1 / 8$ & $2 / 8$ & $1 / 7$ & $0 / 8$ \\
\hline Rectal & $0 / 8$ & $0 / 8$ & $2 / 8$ & $1 / 7$ & $0 / 8$ \\
\hline \multicolumn{6}{|l|}{ In-contact } \\
\hline Tonsillart & ND & ND & ND & ND & $1 / 8$ \\
\hline Oral & ND & $0 / 8$ & $2 / 8$ & $0 / 8$ & $0 / 8$ \\
\hline Nasal & ND & $0 / 8$ & $1 / 8$ & $0 / 8$ & $0 / 8$ \\
\hline Rectal & ND & $0 / 8$ & $0 / 8$ & $0 / 8$ & $0 / 8$ \\
\hline \multicolumn{6}{|c|}{$\begin{array}{l}\text { Data reported as No. of specimens that yielded growth/No. of specimens. } \\
\text { *One inoculated deer euthanatized and necropsied on day } 90 \text { and } 7 \text { on day } \\
\text { 120. Four in-contact deer euthanatized and necropsied on day } 180 \text { and } 4 \text { on } \\
\text { day } 360 \text {. †Tonsillar swab specimens were collected from inoculated deer on } \\
\text { day } 21 \text { and at necropsy and from in-contact deer, at necropsy only. } \neq \text { One inoc- } \\
\text { ulated deer euthanatized on day } 90 \text {. } \\
\text { ND = Not done. }\end{array}$} \\
\hline
\end{tabular}


rate pen on day 150. Mycobacterium bovis was also isolated from hay samples from 1 of these same pens on days 90 and 210. Bacteriologic culture of feces from the other pen yielded $M$ bovis on day 90. At no time was $M$ bovis isolated from water, nor was it isolated from pelleted feed, hay, or feces after day 210.

Necropsy-At necropsy, all inoculated, original in-contact, and new in-contact deer had 1 or more tissues that contained tuberculous lesions. Inoculated deer developed disseminated tuberculosis; specimens from tonsil, lung, and medial retropharyngeal, tracheobronchial, mediastinal, hepatic, and mesenteric lymph nodes most commonly contained tuberculous lesions (Table 2). Other sites affected in inoculated deer included trachea, liver, spleen, kidney, and mandibular, parotid, superficial cervical, iliac, prefemoral, and popliteal lymph nodes. In contrast, the most common tissues containing tuberculous lesions in original incontact deer were lung and tracheobronchial and medial retropharyngeal lymph nodes. Lesions consistent with $M$ bovis infection were not as commonly detected in tonsils and mediastinal, hepatic, and mesenteric lymph nodes from in-contact deer, compared with inoculated deer.

New in-contact deer most commonly developed lesions in lungs and tracheobronchial, mediastinal, medial retropharyngeal and popliteal lymph nodes. Other sites affected in new in-contact deer included those affected in original in-contact deer. Deer in all 3 groups (inoculated, original in-contact, and new incontact) had pulmonary granulomas involving bronchi or bronchioles. Airways were mildly dilated and filled

Table 2-Distribution of affected tissues* in white-tailed deer inoculated with $M$ bovis or infected through contact with inoculated deer (original in-contact) or original in-contact deer (new incontact)

\begin{tabular}{|c|c|c|c|}
\hline Tissue & Inoculated & $\begin{array}{c}\text { Original } \\
\text { in-contact }\end{array}$ & $\begin{array}{c}\text { New } \\
\text { in-contact }\end{array}$ \\
\hline Tonsil & $7 / 8$ & $4 / 8$ & $2 / 4$ \\
\hline Mandibular LN & $2 / 8$ & $1 / 8$ & $2 / 4$ \\
\hline Parotid LN & $3 / 8$ & $2 / 8$ & $1 / 4$ \\
\hline Medial retropharyngeal LN & $8 / 8$ & $6 / 8$ & $3 / 4$ \\
\hline Superficial cervical LN & $3 / 8$ & $1 / 8$ & $2 / 4$ \\
\hline Trachea & $2 / 8$ & $1 / 8$ & $1 / 4$ \\
\hline Lung & $6 / 8$ & $6 / 8$ & $3 / 4$ \\
\hline Tracheobronchial LN & $7 / 8$ & $5 / 8$ & $3 / 4$ \\
\hline Mediastinal LN & $5 / 8$ & $3 / 8$ & $3 / 4$ \\
\hline Liver & $0 / 8$ & $2 / 8$ & $1 / 4$ \\
\hline Spleen & $2 / 8$ & $2 / 8$ & $2 / 4$ \\
\hline Kidney & $0 / 8$ & $1 / 8$ & $1 / 4$ \\
\hline Hepatic LN & $6 / 8$ & $2 / 8$ & $2 / 4$ \\
\hline Mesenteric LN & $6 / 8$ & $3 / 8$ & $2 / 4$ \\
\hline Brain & $0 / 8$ & $1 / 8$ & $1 / 4$ \\
\hline Intercostal muscle & $1 / 4$ & $1 / 4$ & $0 / 4$ \\
\hline Diaphragm & $1 / 4$ & $1 / 4$ & $1 / 4$ \\
\hline Iliac LN & $2 / 8$ & $1 / 8$ & $2 / 4$ \\
\hline Prefemoral LN & $0 / 8$ & $2 / 8$ & $2 / 4$ \\
\hline Popliteal LN & $1 / 8$ & $1 / 8$ & $3 / 4$ \\
\hline \multicolumn{4}{|c|}{$\begin{array}{l}\text { Data reported as No. of necropsy specimens yielding positive results/No. of } \\
\text { specimens. } \\
\text { *Affected tissues were those in which tuberculous lesions containing acid- } \\
\text { fast bacilli were detected during histologic examination or in which } M \text { bovis } \\
\text { was isolated by use of bacteriologic culture techniques. } \\
\text { LN = Lymph node. }\end{array}$} \\
\hline
\end{tabular}

with macrophages, neutrophils, cellular debris, and variable numbers of acid-fast bacilli (Fig 1). Similarly, deer from all 3 groups had tracheal lesions consisting of mucosal and submucosal caseonecrotic granulomas. Lesions were composed of a mixture of granulomatous to suppurative cellular exudate containing variable numbers of acid-fast bacilli (Fig 2). One deer from the original in-contact and 1 from the new in-contact

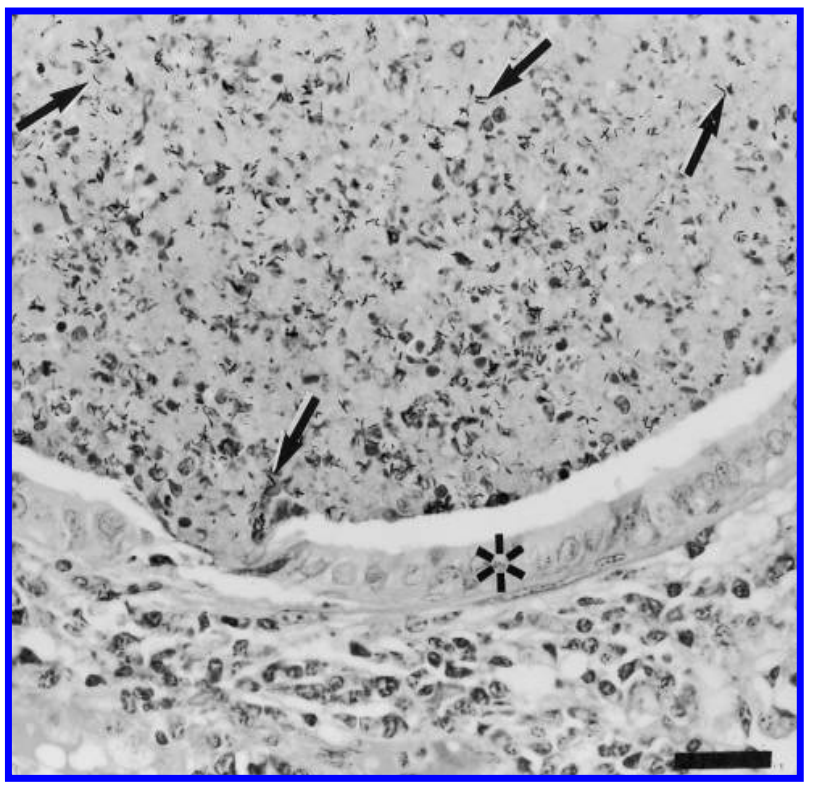

Figure 1-Photomicrograph of a section of lung from a new incontact white-tailed deer (in contact for 180 days with deer that had been in contact for 120 days with deer experimentally infected with Myco bacterium bovis). Bronchiolar lumen is dilated and filled with exudate including numerous acid-fast bacilli (arrows). ${ }^{*}=$ Bronchiolar epithelium. Ziehl-Neelsen acid-fast stain; bar $=35 \mu \mathrm{m}$.

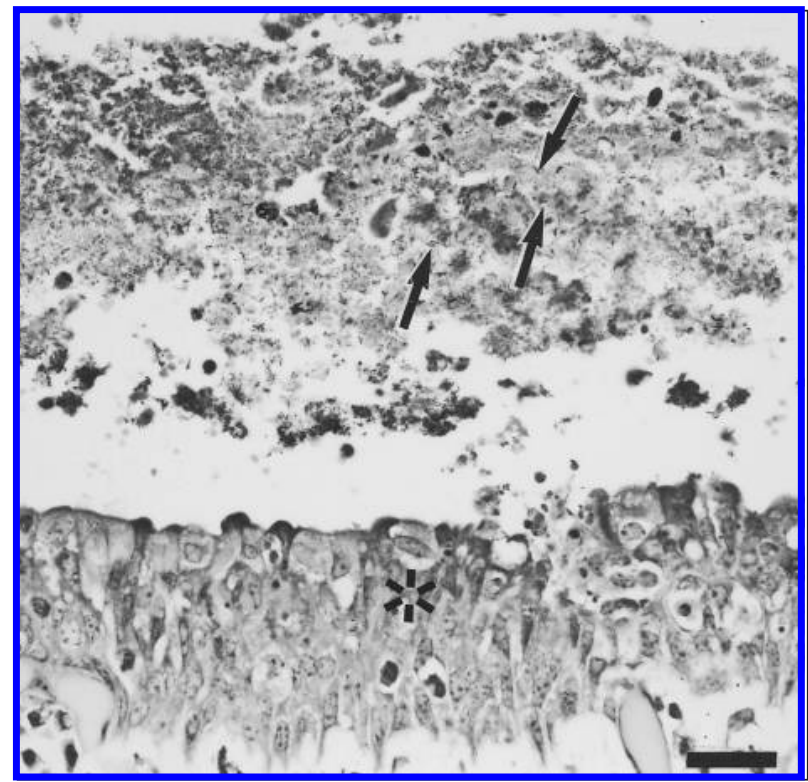

Figure 2-Photomicrograph of a section of trachea from an original in-contact white-tailed deer (deer in contact for 120 days with deer experimentally infected with $M$ bovis). The tracheal lumen contains cellular debris and acid-fast bacilli (arrows) * = Tracheal mucosal epithelium. Ziehl-Neelsen acid fast stain bar $=35 \mu \mathrm{m}$. 


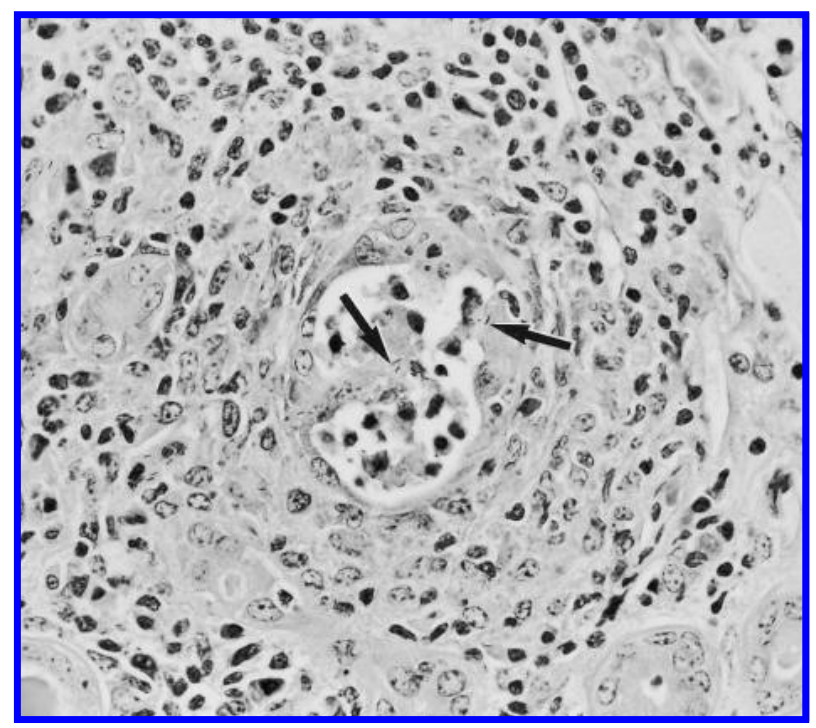

Figure 3-Photomicrograph of a section of kidney from a new in-contact deer. The renal tubular lumen is dilated and contains cellular debris with acid-fast bacilli (arrows). Ziehl-Neelsen acid fast stain; bar $=35 \mu \mathrm{m}$.

groups also had multifocal to coalescing caseonecrotic granulomas within the renal cortex and medulla. Acidfast bacilli were seen within granulomas as well as in macrophages and neutrophils within renal tubules lined by flattened epithelium (Fig 3).

Mycobacterium bovis was isolated from 1 or more tissues from all inoculated, original in-contact, and new in-contact deer. Distribution of tissues from which $M$ bovis was isolated was similar to distribution of tissues with tuberculous lesions. However, $M$ bovis was isolated from the brain of 1 original in-contact deer and 1 new in-contact deer without detection of accompanying lesions. Urine collected at necropsy from 1 of 8 original in-contact deer and feces collected from 1 of 4 new in-contact deer contained $M$ bovis.

\section{Discussion}

Mycobacterium bovis was efficiently transmitted from experimentally infected white-tailed deer to uninfected penmates. After 69 days of co-mingling with experimentally infected deer, all in-contact deer developed delayed-type hypersensitivity (DTH) reactions to purified protein derivative (PPD) of $M$ bovis, and 2 of 8 were shedding $M$ bovis in nasal secretions or saliva. In-contact deer were not euthanatized at this early time; therefore, we do not know whether tuberculous lesions developed after only 69 days. Results of previous studies of experimentally infected cattle indicate that lesions develop in medial retropharyngeal lymph nodes as early as 28 days after intratonsillar inoculation. ${ }^{10}$ In addition, pulmonary lesions have been recognized in calves as early as 7 to 14 days after intranasal inoculation. ${ }^{11,12}$

Mycobacterium bovis was also efficiently transmitted from in-contact infected deer to uninfected penmates. After 100 days of co-mingling with in-contact infected animals, new in-contact deer developed a DTH reaction to $M$ bovis PPD. Shedding of $M$ bovis in saliva or nasal secretions was not as commonly detect- ed from in-contact infected deer as from experimentally infected deer. However, even after experimentally infected deer were euthanatized, (ie, on days 150 and 210) $M$ bovis was isolated from pelleted feed and hay, suggesting that original in-contact deer were shedding $M$ bovis. It is unlikely that the $M$ bovis that contaminated feed was residual contamination from inoculated deer, as these deer had been euthanatized on day 120 . Results of previous studies revealed that $M$ bovis survives only 5 to 14 days in infected tissues during seasons other than winter ${ }^{13}$ and for 7 days on hay at room temperature. $^{e}$ However, $M$ bovis may persist outside during winter in infected tissues for up to 6 weeks ${ }^{13}$ or on feedstuffs at $0 \mathrm{C}$ for up to 16 weeks.

Inhalation and ingestion are the most common modes of $M$ bovis infection. Finding lesions in the lungs and associated lymph nodes of original and new in-contact deer suggested that the route of exposure to $M$ bovis was primarily inhalation. Results of studies indicate that droplet nuclei $<5 \mu \mathrm{m}$ in diameter and containing 1 to 2 bacilli can be inhaled and carried to pulmonary alveoli. ${ }^{14}$ In naturally infected white-tailed deer, the most commonly affected sites are medial retropharyngeal lymph nodes and lungs followed by tracheobronchial and mediastinal lymph nodes. ${ }^{1,7}$ The distribution of lesions detected in the present study does not rule out transmission through contaminated feed as an important means of transmission. Mycobacteria on small feed particles or dust inhaled during feeding would likely result in a lesion distribution pattern indicative of infection through inhalation. Tuberculous lesions of the mesenteric lymph nodes suggested infection through ingestion. However, such lesions may result from either direct ingestion of mycobacteria or swallowing of sputum containing mycobacteria in deer with pulmonary lesions. In transmission studies, using experimentally inoculated or naturally infected calves, a similar pattern of gross lesions was detected in in-contact calves. ${ }^{15,16}$ However, only one third to one fourth of in-contact calves developed tuberculosis. In the present study, transmission of M bovis was $100 \%$. Differences in results between our study and previous studies may be attributable to species differences or differences in source of inoculum, inoculum dose, animal density, or environmental conditions. In the present study, transmission may have been artificially enhanced by the close contact between deer required by housing restrictions of a biosecurity level 3 facility and by dose of inoculum administered. The open environment of more natural surroundings would likely decrease the efficiency of disease transmission.

Shedding of $M$ bovis in nasal secretions and saliva has been documented in experimentally infected white-tailed deer. ${ }^{4}$ Tracheal swab specimens and palatine (oropharyngeal) tonsils from naturally infected white-tailed deer contain $M$ bovis. ${ }^{7}$ Similarly, nasal and tracheal swab specimens and oropharyngeal tonsils from naturally infected red deer (Cervus elaphus) and nasal mucus from naturally infected cattle contain $M$ bovis. ${ }^{17-20}$ Lesions detected in the pulmonary airways, trachea, and kidneys of deer in the present study indicate that coughing, exhalation, or urination are likely 
means of excretion of mycobacteria. Neither feces nor urine from naturally infected white-tailed deer have been shown to contain $M$ bovis. However, feces from naturally infected red deer and experimentally infected cattle does contain $M$ bovis. ${ }^{17,19}$ Mycobacterium bovis was also isolated from urine and feces of experimentally infected badgers (Meles meles). ${ }^{21}$ Our results suggest that feces and urine as well as nasal secretions and saliva from infected deer represent routes by which tuberculosis may be transmitted directly or indirectly to other deer and susceptible hosts.

Our inability to recover $M$ bovis from swab specimens collected from in-contact infected deer at later time points (ie, after day 90) is consistent with results of previous work in experimentally infected cattle. Cattle experimentally infected by intranasal inoculation shed $M$ bovis intermittently, especially in later stages of the experiment. ${ }^{15,16}$ Intermittent shedding could result in the inability to isolate $M$ bovis from swab specimens collected every 30 days, as was done in the present study.

In northern Michigan, $M$ bovis infection is endemic in free-ranging white-tailed deer, and feeding of deer during the winter has been incriminated as a means of maintaining infection. ${ }^{1}$ Large numbers of deer around feeding sites provide opportunity for close contact and aerosol transmission of $M$ bovis. In addition, such an environment may allow infected deer to contaminate feed consumed by uninfected deer or other susceptible animals. In Switzerland, naturally infected roe deer (Capreolus capreolus) are suspected to have infected domestic cattle through contaminated feed. ${ }^{22}$ Cattle have also been shown to become infected with $M$ bovis by contact with feces or urine from infected badgers. ${ }^{21}$ Under appropriate conditions, $M$ bovis may persist in the environment for weeks or months. ${ }^{13,23,24}$ Therefore, the possibility of transmission through both direct and indirect contact must be considered. Wildlife managers in tuberculosis-endemic areas should discourage practices that promote the unnatural gathering of deer, because such gatherings enhance both direct and indirect transmission of $M$ bovis and makes disease control difficult and eradication unlikely.

\footnotetext{
${ }^{a}$ Whipple DL, Jarnagin JL, Payeur JB. DNA fingerprinting of Mycobacterium bovis isolates from animals in northeast Michigan (abstr), in Proceedings. IX Int Symp World Assoc Vet Lab Diagnosticians 1999;83.

bDeer and elk complete feed 55P3, Purina Mills, St Louis, Mo.

${ }^{c}$ Cytobrush, Puritan Medical Products, Guilford, Me.

dSleepaway, Fort Dodge Laboratories, Fort Dodge, Iowa.

'Whipple DL, Palmer MV. Survival of Mycobacterium bovis on feeds used for baiting white-tailed deer (Odocoileus virginianus) in Michigan (abstr), in Proceedings. 49th Annu Wildl Dis Assoc $2000 ; 21$.
}

\section{References}

1. Schmitt SM, Fitzgerald SD, Cooley TM, et al. Bovine tuberculosis in free-ranging white-tailed deer from Michigan. $L$ Wildl Dis 1997:33:749-758.

2. Frye GH. Bovine tuberculosis eradication: the program in the United States. In: Thoen CO, Steele JH, eds. Mycobacterium bovis infection in animals and humans. Ames, Iowa: Iowa State University Press, 1995;119-129.

3. Whipple DL, Meyer RM, Berry DF, et al. Molecular epidemiology of tuberculosis in wild white-tailed deer in Michigan and elephants, in Proceedings. U S Anim Health Assoc 1997;543-546.

4. Palmer MV, Whipple DL, Olsen SC. Development of a model of natural infection with Mycobacterium bovis in white-tailed deer. I Wildl Dis 1999;35:450-457.

5. Centers for Epidemiology and Animal Health Evaluation of tuberculin testing in cervidae. Fort Collins, Colo: USDA, Animal and Plant Health Inspection Service,Veterinary Services, 1996;7-8.

6. USDA. Bovine tuberculosis eradication uniform methods and rules. Washington, DC: US Government Printing Office, 1999;4.

7. Palmer MV, Whipple DL, Payeur JB, et al. Naturally occur-

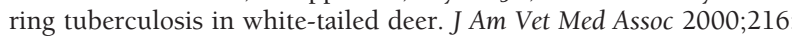
1921-1924.

8. Payeur JB, Jarnagin JL, Marquardt JG. Laboratory methods in veterinary mycobacteriology for the isolation and identification of mycobacteria. Ames, Iowa: USDA, Animal and Plant Health Inspection Service, National Veterinary Services Laboratories, 1992;5-54.

9. Sheehan DC, Hrapchak BB. Microorganisms. In: Theory and practice of histotechnology. 2nd ed. St Louis: Mosby Publishing Co, 1980;233-251.

10. Palmer MV, Whipple DL, Rhyan JC, et al. Granuloma development in cattle after intratonsilar inoculation with Mycobacterium bovis. Am I Vet Res 1999;60:310-315.

11. Cassidy JP, Bryson DG, Pollock JM, et al. Early lesion formation in cattle experimentally infected with Mycobacterium bovis. I Comp Pathol 1998;119:27-44.

12. Cassidy JP, Bryson DG, Pollock JM, et al. Lesions in cattle exposed to Mycobacterium bovis-inoculated calves. L Comp Pathol 1999;121:321-327.

13. Tanner M, Michel AL. Investigation of the viability of $M$ bovis under different environmental conditions in the Kruger National Park. Onderstepoort I Vet Res 1999;66:185-190.

14. Riley RL, Mills CC, Nyka W, et al. Aerial dissemination of pulmonary tuberculosis. Am J Hyg 1959;70:185-196.

15. Neill SD, Hanna J, O'Brien JJ, et al. Transmission of tuberculosis from experimentally infected calves to in-contact calves. Vet Rec 1989;124:269-271.

16. Costello E, Doherty ML, Monaghane ML, et al. A study of cattle-to-cattle transmission of Mycobacterium bovis. Vet I 1998;155: 245-250.

17. Lugton IW, Wilson PR, Morris RS, et al. Epidemiology and pathogenesis of Mycobacterium bovis infection of red deer (Cervus elaphus) in New Zealand. N Z Vet I 1998;46:147-156.

18. de Kantor IN, Bioch D, Roswurm JD. Mycobacteria isolated from nasal secretions of tuberculin test reactor cattle. Am I Vet Res 1973;39:1233-1234.

19. Neill SD, O'Brien JJ, McCracken RM. Mycobacterium bovis in the anterior respiratory tracts in the heads of tuberculin-reacting cattle. Vet Rec 1988;122:184-186.

20. Neill SD, Hanna J, Mackie DP, et al. Isolation of Mycobacterium bovis from the respiratory tracts of skin-negative cattle. Vet Rec 1992;131:45-47.

21. Little TWA, Naylor PF, Wilesmith JW. Laboratory study of Mycobacterium bovis infection in badgers and calves. Vet Rec 1982;111:550-557.

22. Bischofberger VA, Nabholz A. Tuberkulöes wild als ursache von neuinfektionen in rindviehbeständen. Schweizer Archiv für Tierheilkunde 1964;106:759-777.

23. Duffield BJ, Young DA. Survival of Mycobacterium bovis in defined environmental conditions. Vet Microbiol 1985;10:193-197.

24. Jackson R, de Lisle GW, Morris RS. A study of the environmental survival of Mycobacterium bovis on a farm in New Zealand. N 